\title{
COVERS OF DEHN FILLINGS ON ONCE-PUNCTURED TORUS BUNDLES
}

\author{
MARK D. BAKER
}

(Communicated by Frederick R. Cohen)

\begin{abstract}
Let $M$ be a compact, orientable 3-manifold that fibers over $S^{1}$ with fiber a once-punctured torus, $T_{0}$, and characteristic homeomorphism $h: T_{0} \rightarrow T_{0}$. We prove that for certain characteristic homeomorphisms, most Dehn fillings on $M$ yield manifolds with virturally $\mathrm{Z}$-representable fundamental groups.
\end{abstract}

\section{INTRODUCTION AND STATEMENT OF RESULTS}

Let $M$ be a compact, orientable 3-manifold that fibers over $S^{1}$ with fiber a once-punctured torus, $T_{0}$, and characteristic homeomorphism $h: T_{0} \rightarrow T_{0}$. In this paper we investigate the manifolds obtained from $M$ by Dehn fillingattaching a solid torus to the torus boundary of $M$ to obtain a closed 3manifold. We prove that for certain characteristic homeomorphisms, most Dehn fillings on $M$ yield manifolds with virtually Z-representable fundamental groups.

Let $D_{x}$ (resp. $D_{y}$ ) denote the left-handed Dehn twist about the curve $x$ (resp. curve $y$ ) in $T_{0}$ (see Figure 1). Then any orientation preserving homeomorphism

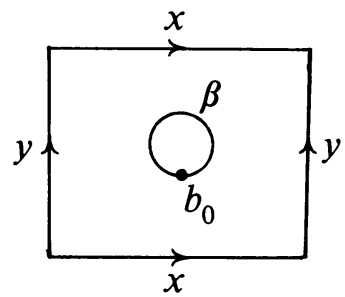

FIGURE 1

$h: T_{0} \rightarrow T_{0}$ can be represented up to isotopy by a composition of the Dehn twists $D_{x}$ and $D_{y}$. We prove:

Received by the editors May 20, 1988 and, in revised form, October 16, 1988.

1980 Mathematics Subject Classification (1985 Revision). Primary 57M10. 57N10. 
Theorem. Let $M$ be a once-punctured torus bundle over $S^{1}$ with characteristic homeomorphism $h$ of the form $D_{x}^{r_{1}} \circ D_{y}^{s_{1}} \circ \cdots \circ D_{x}^{r_{k}} \circ D_{y}^{s_{k}}$ and let $m=$ g.c.d. $\left(r_{1}, \ldots, r_{k}\right), n=$ g.c.d. $\left(s_{1}, \ldots, s_{k}\right)$. Then

(a) If $m \geq 2, n \geq 2, m n \geq 8$ but $m n \neq 9$, then all Dehn fillings on $M$ of the form $(\mu, \lambda),|\lambda| \neq 1$ yield manifolds with virtually Z-representable fundamental groups.

(b) If $m \geq 2, n \geq 2$ and $m n=6$ or 9, then all Dehn fillings on $M$ of the form $(\mu, \lambda)$ with $|\lambda| \neq 1$ or 2 yield manifolds with virtually Z-representable fundamental groups.

Remarks. 1. The $(0,1)$ filling on any punctured surface bundle over $S^{1}$ yields a manifold with positive first Betti number. Hence the cases not covered by our theorem are $(\mu, \lambda)=(n, 1), n \neq 0$, as well as $(\mu, \lambda)=(n, 2), n \neq 0$ in part (b).

2. Other results on the virtual Z-representability of 3-manifold groups can be found in $\left[\mathrm{B}_{1}\right],\left[\mathrm{B}_{2}\right],\left[\mathrm{H}_{1}\right],\left[\mathrm{H}_{2}\right]$, and [M].

Dehn filling on hyperbolic torus bundles gave the first example of hyperbolic non-Haken 3-manifolds. Indeed, it follows from $[\mathrm{F}, \mathrm{H}],[\mathrm{C}, \mathrm{J}, \mathrm{R}]$ and $[\mathrm{T}]$ that all but finitely many Dehn fillings on hyperbolic once-punctured torus bundles yield manifolds that are hyperbolic but contain no incompressible surfaces. However for the hyperbolic torus bundles in our theorem, most Dehn fillings yield manifolds that are virturally Haken.

\section{DEFINITIONS AND OUTLINE OF PROOF}

Relevant background material on torus bundles and Dehn twist homeomorphisms can be found in $\left[\mathrm{H}_{2}\right]$ and [R].

By Dehn filling on a 3-manifold $M$ with respect to a simple loop in a boundary torus we mean attaching a solid torus to $\partial M$ so that this loop bounds a meridional disk in the solid torus.

We say that $M$ has a virtually Z-representable fundamental group if $\pi_{1}(M)$ contains a finite index subgroup that maps epimorphically to $\mathbf{Z}$, or equivalently if there is a finite cover $\widetilde{M} \rightarrow M$ with rank $H_{1}(\widetilde{M}) \geq 1$. In particular, if $M$ is compact, orientable and irreducible, then the virtual Z-representability of $\pi_{1}(M)$ implies that the above cover $\widetilde{M}$ is a Haken manifold.

Now let $M$ be a once-punctured torus bundle with characteristic homeomorphism $h: T_{0} \rightarrow T_{0}$ which is the identity on $\partial T_{0}$. Choose a base point $b_{0}$ in $\partial T_{0}$ and consider the loops $\alpha=b_{0} \times[0,1] / \sim$ and $\beta=\partial T_{0}$ in $\partial M$ (see Figure 1). Denote by $M(\mu, \lambda)$ the manifold obtained from Dehn filling on $M$ with respect to the loop $\alpha^{\mu} \beta^{\lambda}$ in $\partial M$.

For a torus bundle, $M$, with characteristic homeomorphism, $h$, and Dehn filling parameters $(\mu, \lambda)$ as in the theorem, we prove our result by constructing a finite cover $\widetilde{M} \rightarrow M$ with the following two properties:

(i) The cover $\widetilde{M} \rightarrow M$ extends to a cover $N \rightarrow M(\mu, \lambda)$ by Dehn filling on $\widetilde{M}$ and on $M$. 
(ii) $\operatorname{Rank} H_{1}(\widetilde{M})>$ number of components of $\partial \widetilde{M}$.

Property (ii) guarantees that any manifold obtained by Dehn filling on $\widetilde{M}$ (hence $N$ ) has positive first Betti number. Hence $M(\mu, \lambda)$ has virtually $\mathbf{Z}$ representable fundamental group.

The cover $\widetilde{M}$ is gotten by constructing a cover $\pi: F \rightarrow T_{0}$ to which $h$ lifts to $\tilde{h}: F \rightarrow F$. Then the mapping torus, $\widetilde{M}$, of the pair $(F, \widetilde{h})$ is the desired cover of $M$.

\section{Proof of case (a) of theorem}

Recall that the characteristic homeomorphism, $h$, of $M$ is of the form $D_{x}^{r_{1}} \circ D_{y}^{s_{1}} \circ \ldots \circ D_{x}^{r_{k}} \circ D_{y}^{s_{k}}$ where g.c.d. $\left(r_{1}, \ldots, r_{k}\right)=m$ and g.c.d. $\left(s_{1}, \ldots, s_{k}\right)=n$. We can assume that $m \geq 2$ and $n \geq 4$. Denote by $\pi: S \rightarrow T_{0}$ the $m n$-fold cover corresponding to the kernel of the map $\theta: \pi_{1}\left(T_{0}\right) \cong \mathbf{Z} \times \mathbf{Z} \rightarrow \mathbf{Z} / m \times \mathbf{Z} / n$ where $\theta([x])=(1,0)$ and $\theta([y])=(0,1) . S$ is a torus with $m n$ punctures, $\left\{\widetilde{\beta}_{i}\right\}$, pictured in Figure 2 .

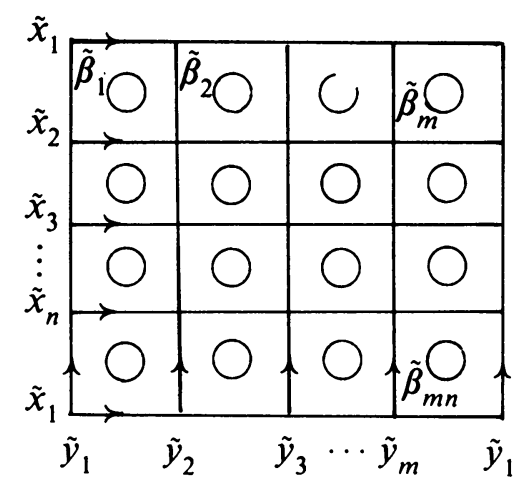

FIGURE 2

Lemma 3.1. The homeomorphism $h: T_{0} \rightarrow T_{0}$ lifts to a homeomorphism $g$ : $S \rightarrow S$.

Proof. The loop $x$ (resp. $y$ ) in $T_{0}$ lifts to $n$ loops $\tilde{x}_{1}, \ldots, \tilde{x}_{n}$ (resp. $m$ loops $\tilde{y}_{1}, \ldots, \tilde{y}_{m}$ ) in $S$ that project $m$ to 1 onto $x$ (resp. $n$ to 1 onto $y$ ). Since $m \mid r_{i}$ and $n \mid s_{i}$, the Dehn twists $D_{x}^{r_{i}}$ lift to Dehn twists about the lifts, $\left\{\tilde{x}_{i}\right\}$, of $x$ and the Dehn twists $D_{y}^{s_{i}}$ lift to Dehn twists about the lifts, $\left\{\tilde{y}_{i}\right\}$, of $y$. Hence $h$ lifts to a homeomorphism $g: S \rightarrow S$.

We begin with the $(1,0)$ Dehn filling on $M$-the case $\lambda=0$. Let $\widetilde{M}$ denote the mapping torus of $(S, g)$. By construction, the "meridian" loop $\alpha=b_{0} \times[0,1] / \sim$ in $\partial M$ lifts to $m n$ loops $\tilde{\alpha}_{1}, \ldots, \tilde{\alpha}_{m n}$-indexed so that the loops $\tilde{\alpha}_{i}, \widetilde{\beta}_{i}$ lie on the $i$ th boundary torus of $\widetilde{M}$ (cf. Figure 2). 
Lemma 3.2. The cover $\widetilde{M} \rightarrow M$ extends to a cover $N \rightarrow M(1,0)$ by Dehn filling on $\widetilde{M}$ and $M$ with respect to the curves $\left\{\tilde{\alpha}_{i}\right\}$ in $\partial \widetilde{M}$ and $\alpha$ in $\partial M$.

Lemma 3.3. Rank $H_{1}(N) \geq 1$.

Proof. There are relations in $H_{1}(\widetilde{M})$ between the lifts $\left\{\tilde{\alpha}_{i}\right\}$ of $\alpha$. For example $\left[\tilde{\alpha}_{2}\right]-\left[\tilde{\alpha}_{1}\right]=\left[\tilde{\alpha}_{m+2}\right]-\left[\tilde{\alpha}_{m+1}\right]$. Thus Dehn filling with respect to the loops $\left\{\tilde{\alpha}_{i}\right\}$ yields a manifold $N$ with positive first Betti number. Indeed, one computes $\left[\tilde{\alpha}_{j}\right]-\left[\tilde{\alpha}_{i}\right]$ as follows. Let $\sigma_{i j}$ be a simple path in $S$ from the point $\widetilde{b}_{i}=\tilde{\alpha}_{i} \cap S$ to the point $\widetilde{b}_{j}=\tilde{\alpha}_{j} \cap S\left(\widetilde{b}_{i} \in \widetilde{\beta}_{i}\right.$ is the lift of the basepoint $b_{0} \in \beta$ in $\left.T_{0}\right)$. Then the disk $\sigma_{i j} \times I \subset S \times I$ provides the relation $\left[\tilde{\alpha}_{j}\right]-\left[\tilde{\alpha}_{i}\right]=\left[g\left(\sigma_{i j}\right) * \sigma_{i j}^{-1}\right]$ in $H_{1}(\widetilde{M})$, where $*$ denotes composition of paths.

Now $\sigma_{12}$ and $\sigma_{m+1, m+2}$ can be chosen so that they both intersect the Dehn twist curve $\tilde{y}_{2}$ exactly once and have zero intersection with all other Dehn twist curves $\tilde{x}_{i}, \tilde{y}_{i}$ of the homeomorphism $g: S \rightarrow S$. Thus it follows that $\left[g\left(\sigma_{12}\right) * \sigma_{12}^{-1}\right]=\left[g\left(\sigma_{m+1, m+2}\right) * \sigma_{m+1, m+2}^{-1}\right]$ in $H_{1}(\widetilde{M})$.

Now consider the $(\mu, \lambda)$ Dehn filling on $M$ where $|\lambda|=d \geq 2$. We construct the desired cover $\pi: F \rightarrow T_{0}$ by gluing together $d$ copies of the surface $S$ along certain cuts. Let $S_{1}, \ldots, S_{d}$ be copies of $S$ and make four vertical cuts $\left\{\tau_{j}\right\}$ on each $S_{i}$ as pictured in Figure 3. Glue the left edges of the cuts $\tau_{1}, \tau_{3}$ in $S_{i}$ to the right edges of the same cuts in $S_{i+1}(\bmod d)$ and glue the left edges of the cuts $\tau_{2}, \tau_{4}$ in $S_{i}$ to the right edges of the same cuts in $S_{i-1}(\bmod d)$. Denote this surface by $F . F$ has genus $4 d-3$ and $d m n-8 d+8$ punctures. Then

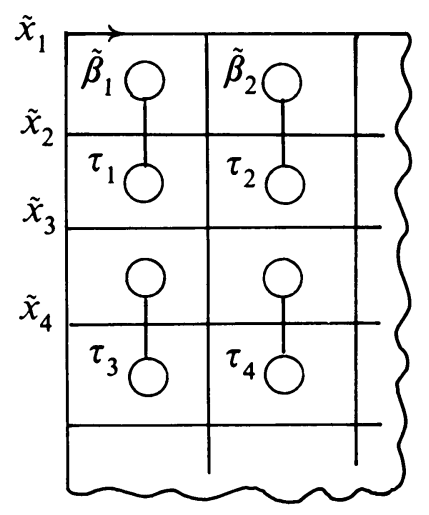

FigURE 3

Lemma 3.4. $\pi: F \rightarrow T_{0}$ is a (non-regular) covering, where $\left.\pi\right|_{s_{i}}$ is defined to be $\pi_{i}: S_{i} \rightarrow T_{0}$. 
Lemma 3.5. The homeomorphism $h: T_{0} \rightarrow T_{0}$ lifts to a homeomorphism $\tilde{h}$ : $F \rightarrow F$.

Proof. $F$ was constructed so that the loop $x$ (resp. $y$ ) in $T_{0}$ lifts to $d n$ loops $\tilde{x}_{1}, \ldots, \tilde{x}_{d n}$ (resp. $d m$ loops $\tilde{y}_{1}, \ldots \tilde{y}_{d m}$ ) in $F$ which project $m$ to 1 onto $x$ (resp. $n$ to 1 onto $y$ ). Thus the Dehn twists $D_{x}^{r_{i}}$ (resp. $D_{y}^{s_{i}}$ ) in $T_{0}$ lift to Dehn twists about the loops $\left\{\tilde{x}_{i}\right\}$ (resp. $\left\{\tilde{y}_{i}\right\}$ ) in $F$. Consequently $h$ lifts to $\tilde{h}: F \rightarrow F$.

Denote by $\widetilde{M}$ the mapping torus of $(F, \widetilde{h})$. By construction the loops $\alpha$ and $\beta^{\lambda}$ in $\partial M$ lift to loops in the boundary tori of $\widetilde{M}$, so the loop $\alpha^{\mu} \beta^{\lambda}$ lifts to loops in $\partial \widetilde{M}$. Choose one lift in each component of $\partial \widetilde{M}$ and label them $\left\{c_{i}\right\}$.

Lemma 3.6. The cover $\widetilde{M} \rightarrow M$ extends to a cover $N \rightarrow M(\mu, \lambda)$ by Dehn filling on $\widetilde{M}$ and $M$ with respect to the curves $\left\{c_{i}\right\}$ in $\partial \widetilde{M}$ and $\alpha^{\mu} \beta^{\lambda}$ in $\partial M$.

Now all that remains to show is

Lemma 3.7. Rank $H_{1}(N) \geq 1$.

Proof. As pointed out in section 2 , it suffices to show that rank $H_{1}(\widetilde{M})>$ number of boundary tori of $\widetilde{M}$.

Since $\widetilde{M}$ is the mapping torus of $(F, \widetilde{h})$, we have $H_{1}(\widetilde{M}) \cong \mathbf{Z} \oplus$ Coker $\left(\widetilde{h}_{*}-\right.$ Id: $\left.H_{1}(F) \rightarrow H_{1}(F)\right)$ where the first factor is generated by any loop (e.g. a lift of $\alpha)$ transverse to $F$. As rank Coker $\left(\widetilde{h}_{*}-\right.$ Id) is equal to the rank of the subgroup of $H_{1}(F)$ fixed by $\widetilde{h}_{*}$ and the boundary components of $F$ are fixed by $\tilde{h}$, it follows that

$$
\operatorname{rank} H_{1}(\widetilde{M}) \geq \text { number of boundary components of } \widetilde{M}
$$

since the boundary components of $F$ and $\widetilde{M}$ are in 1-1 correspondence and there is one relation in $H_{1}(F)$ between the components of $\partial F$ given by the surface, $F$.

Thus we need only exhibit one fixed class in $H_{1}(F)$ that is not homologous to a sum of boundary curves of $F$. A portion of the surface $F$ is shown in Figure 4. (Recall the gluing of the surfaces $S_{i}$ along the cuts $\left\{\tau_{j}\right\}$ ). The class $[\gamma]+[\delta]$ in $H_{1}(F)$ corresponding to the curves $\gamma, \delta$ is fixed by $\widetilde{h}_{*}$, since $\gamma$ and $\delta$ each intersect with opposite orientations the same two Dehn twist curves. Finally $[\gamma]+[\delta]$ is not homologous in $H_{1}(F)$ to a sum of boundary curves of $F$ since $\gamma$ and $\delta$ have nontrivial intersection with the curves $\sigma$ and $\rho$ while all the boundary components of $F$ have null intersection with $\sigma$ and $\rho$.

This concludes the proof case (a) of the theorem.

Remark. The above arguments generalize to show that rank $H_{1}(N) \geq\left[\frac{m}{2}\right]\left[\frac{n}{4}\right]$. 

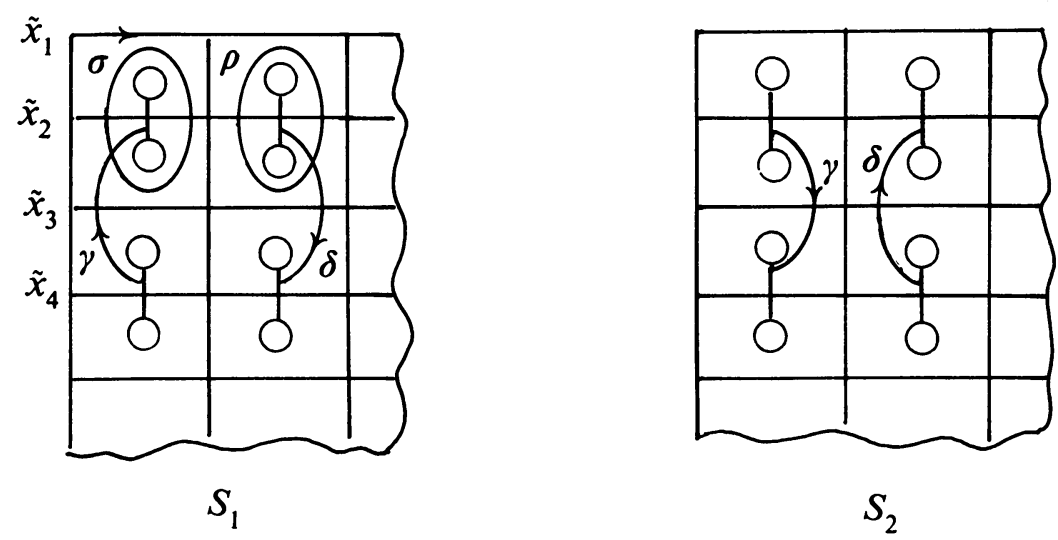

FIGURE 4

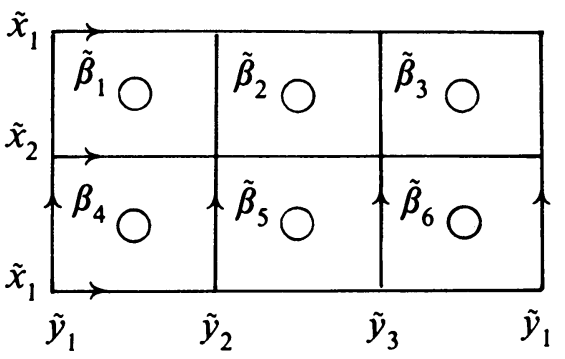

FIGURE 5

\section{Proof of CASe (b) of theorem}

We assume that $m=3$ and $n=2$. The proof when $m=n=3$ is analogous.

The punctured torus $T_{0}$ has a 6-fold cover, $S$, corresponding to the kernel of the homomorphism $\theta: \pi_{1}\left(T_{0}\right) \cong \mathbf{Z} \times \mathbf{Z} \rightarrow \mathbf{Z} / 3 \times \mathbf{Z} / 2$ where $\theta([x])=(1,0)$ and $\theta([y])=(0,1)$ (see Figure 5).

The homeomorphism $h: T_{0} \rightarrow T_{0}$ lifts to a homeomorphism $g: S \rightarrow S$. Denote by $\widetilde{M}$ the mapping torus $(S, g)$. As in section 3 we have:

Lemma 4.1. The cover $\widetilde{M} \rightarrow M$ extends to a cover $N \rightarrow M(1,0)$ by Dehn filling on $M$ with respect to the curve $\alpha$ and on $\widetilde{M}$ with respect to the lifts $\left\{\tilde{\alpha}_{i}\right\}$ of $\alpha$ in $\partial \widetilde{M}$.

Lemma 4.2. Rank $H_{1}(N) \geq 1$.

Now consider the $(\mu, \lambda)$ Dehn filling on $M$ where $|\lambda|=d \geq 3$. We construct the desired cover $F \rightarrow T_{0}$ by gluing together $d$ copies of $S$ along three vertical cuts $\left\{\tau_{j}\right\}$ as pictured in Figure 6. Glue the left edges of the cuts $\tau_{1}$ and $\tau_{2}$ on $S_{i}$ to the right edges of the same cuts on $S_{i+1}(\bmod d)$. Glue the left edge of 
the cut $\tau_{3}$ on $S_{i}$ to the right edge of the same cut on $S_{i-2}(\bmod d)$. As before, call this surface $F$. The cover $F \rightarrow T_{0}$ is $6 d$-fold and the homeomorphism $h: T_{0} \rightarrow T_{0}$ lifts to a homeomorphism $\widetilde{h}: F \rightarrow F$. Let $\widetilde{M}$ denote the mapping torus of $(F, \widetilde{h})$. By construction the loop $\alpha^{\mu} \beta^{\lambda}$ in $\partial M$ lifts to loops in the boundary tori of $\widetilde{M}$.

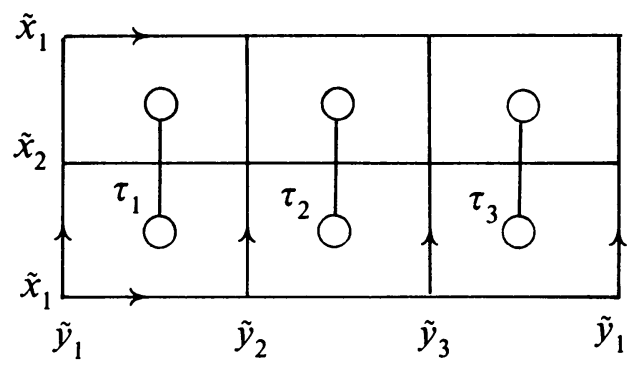

FIGURE 6

Lemma 4.3. The cover $\widetilde{M} \rightarrow M$ extends to a cover $N \rightarrow M(\mu, \lambda)$ by Dehn filling on $M$ with respect to $\alpha^{\mu} \beta^{\lambda}$ and on $\widetilde{M}$ with repsect to lifts of $\alpha^{\mu} \beta^{\lambda}$ in $\partial \widetilde{M}$.

Lemma 4.4. Rank $H_{1}(N) \geq 1$.

Proof. Consider the curves $\gamma$ and $\delta$ on $F$ in Figure 7. The class $[\gamma]+[\delta]$ in $H_{1}(F)$ is fixed by $\widetilde{h}_{*}$ and is not homologous to a sum of boundary curves of $F$.

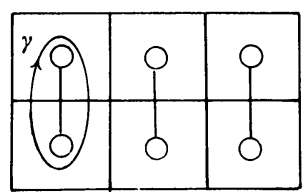

$S_{1}$

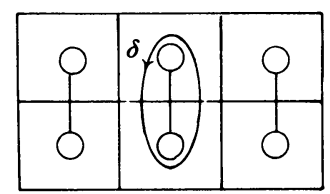

$S_{2}$

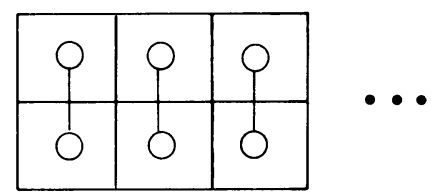

$S_{3}$

FIGURE 7

\section{REFERENCES}

[B 1 ] M. Baker, On certain branched cyclic covers of $S^{3}$, Geometry and Topology (Proc. Univ. Georgia Top. Conf., 1985), Marcel Dekker, New York, 1987, 43-46.

$\left[\mathrm{B}_{2}\right]$, The virtual Z-representability of certain 3-manifold groups, Proc. Amer. Math. Soc. 103 (1988), 996-998. 
[C,J,R] M. Culler, W. Jaco, and H. Rubinstein, Incompressible surfaces in once-punctured torus bundles, Proc. London Math. Soc. (3) 45 (1982), 385-419.

$[\mathrm{F}, \mathrm{H}] \mathrm{W}$. Floyd and A. Hatcher, Incompressible surfaces in punctured torus bundles, Topology and its Applications 13 (1982), 263-282.

$\left[\mathrm{H}_{1}\right]$ J. Hempel, Orientation reversing involutions and the first Betti number for finite coverings of 3-manifolds, Invent. Math. 67 (1982), 133-142.

$\left[\mathrm{H}_{2}\right]$ _ Coverings of Dehn fillings of surface bundles, Topology Appl. 24 (1986), 157-170.

[M] S. Morita, Finite coverings of punctured torus bundles and the first betti number, preprint.

[R] D. Rolfsen, Knots and Links, Publish or Perish Inc., 1976.

[T] W. Thurston, The geometry and topology of 3-manifolds, Xeroxed notes, Princeton University.

Department of Mathematics, Vanderbilt University, Nashville, Tennessee 37235 